\title{
Alta frecuencia de serología positiva contra toxocara en un hospital pediátrico del Perú
}

\author{
High prevalence of toxocara positive serology in a Peruvian pediatric hospital
}

\author{
Edwin Miranda-Choque ${ }^{1 a}$ \\ Instituto Nacional de Salud del Niño, Lima, Perú. \\ a Médico Infectólogo.
}

\begin{abstract}
Resumen
Introducción: Las enfermedades zoonóticas trasmitidas por animales domésticos pueden ser consideradas como problema en salud pública en países en desarrollo. Objetivo: Determinar la frecuencia de serología positiva contra toxocara en niños con sospecha clínica de toxocariasis. Diseño: Estudio descriptivo trasversal. Institución: Instituto Nacional de Salud del Niño (INSN), Lima, Perú. Participantes: Niños con serología positiva para toxocara. Métodos: Se estudió los casos positivos para toxocara por serología, en el periodo 2007 a 2010. La información se obtuvo de las historias clínicas. Principales medidas de resultados: Frecuencia y características de los casos seropositivos contra toxocara. Resultados: De 242 casos con sospecha de toxocara, $148(61,2 \%)$ fueron seropositivos contra toxocara, siendo la media de edad 6,8 (DE 3,8) años; el grupo etario de 2 a 10 correspondió a 69,6\%; el motivo de consulta fue la eosinofilia en 70,9\%. Conclusiones: El INSN atiende niños con una alta frecuencia de serologia positiva contra toxocara, en pacientes sospechosos por esta enfermedad; esto ocurre principalmente en los niños de 2 a 10 años, hallándose aumento de los eosinófilos.
\end{abstract}

Palabras clave: Toxocara, serología, niños

Abstract

Introduction: Zoonotic diseases transmitted by domestic animals may be considered a public health problem in developing countries. Objective: To determine the frequency of positive toxocara serology in clinically suspected children. Design: Descriptive, cross-sectional study. Setting: Instituto Nacional de Salud del Niño (INSN), Lima, Peru. Participants: Children with positive toxocara serology. Methods: Clinical records of children with suspicion of toxocara infection during the period 2007-2010 were included. Main outcome measures: Frequency and characteristics of toxocara seropositive cases. Results: From the 242 cases studied $61.2 \%$ (148) were toxocara seropositive, with average age 6.8 (SD 3.8) years; the group 2-10 years-old represented $69.6 \%$ of the total; eosinophilia was the main reason for consultation in $70.9 \%$. Conclusions: High frequency of toxocara positive serology is found at INSN in suspicious patients, especially in children 2-10 years-old with eosinophilia.

Keywords: Toxocara, serology, children.

An Fac med. 2014;75(3):223-6 / doi: http://dx.doi.org/10.15381/anales.v75i3.9774

\section{INTRODUCCIÓN}

La parasitosis por toxocara es un problema frecuente en la salud pública mundial, especialmente en los países subdesarrollados, donde existen pocos estudios que demuestren la frecuencia real de esta afección en nuestros niños ${ }^{(1,2)}$. Esta enfermedad es una zoonosis que afecta al hombre y es causada principalmente por el Toxocara canis $^{(1,2)}$. La infestación se origina al ingerir huevos de T. canis que se encuentran en el suelo y por ello es frecuente la infestación en niños pequeños que juegan con arena ${ }^{(1-3)}$. El toxocara, en su vida adulta, no se encuentra en el intestino humano; por lo tanto, no es posible hallar huevos del parásito en materia fecal. Por ello, el diagnóstico se basa en el antecedente de exposición a huevos de toxocara, hallazgos de larvas en las biopsias y la detección de anticuerpos contra los antígenos de T. canis empleando el método de inmunoenzimoanálisis o ELISA, de acuerdo a recomendaciones de organismos internacionales, por tener buena sensibilidad y especificidad ${ }^{(4-6)}$.
La gravedad de los síntomas depende de la cantidad y la localización de las larvas; así también, el grado de respuestas alérgicas. Habitualmente, la presentación clínica denominada larva migratoria visceral (LMV) afecta a los niños de 1 a 4 años con antecedente de pica. Es causada por la migración de larvas a través de los órganos internos, ocasionando una variedad de síntomas, incluyendo fatiga, anorexia, disminución del peso, neumonía, fiebre, tos, broncoespasmo, dolor abdominal, cefalea, rash y ocasionalmente convulsiones; puede presentarse también de manera subclí- 
nica, denominada toxocariasis encubierta ${ }^{(7)}$. Otra forma de presentación importante es la larva migratoria ocular (LMO), cuando sobreviene invasión ocular (endooftalmitis o granulomas retinianos) ${ }^{(8)}$.

Los estudios epidemiológicos demuestran su distribución mundial; por ello podemos encontrarlo en países desarrollados y en desarrollo, en países con climas templados a tropicales ${ }^{(7)}$, en población urbana y rural ${ }^{(9)}$; se le relaciona con la tenencia de perros, hábitos alimenticios inadecuados y labores ocupacionales ${ }^{(10)}$.

El Dr. Maguiña, en 1991, realizó el primer reporte de casos de toxocara en la forma LMV en Perú ${ }^{(11)}$. Pero, el primer estudio seroepidemiológico en comunidad lo realizaron Roldán y col, quienes hallaron frecuencia de anticuerpos antitoxocara de $31,1 \%$ en una zona norte de la ciudad de Lima ${ }^{(12)}$.

El presente estudio tiene como objetivo determinar la frecuencia de serología positiva contra toxocara en los niños con sospecha de toxocariasis que acudieron al Instituto Nacional de Salud del Niño (INSN) durante el periodo 2007 a 2010.

\section{MÉTODOS}

El presente es un estudio descriptivo trasversal de pacientes menores de 18 años, atendidos entre 2007 y 2010, con sospecha de toxocariasis. Se realizó el examen serológico de ELISA (enzyme linked immunosorbent assay) para toxocara, en el laboratorio del INSN. La información se obtuvo de las historias clínicas. Consideramos serología positiva para toxocara la detección de anticuerpos específicos anti-toxocara IgG por ELISA (ELISA novum, Novum Diagnostika GmbH, Alemania), con valores mayores de 1,1 .

Se obtuvo información del hemograma, hematocrito, estado nutricional y motivo de consulta, para realizar un análisis descriptivo, mediante frecuencias y valores de tendencia central y dispersión.
Todos los procedimientos del presente estudio preservaron la integridad y los derechos fundamentales de los sujetos a investigación, de acuerdo con los lineamientos de las buenas prácticas clínicas y de ética en investigación biomédica.

\section{RESULTADOS}

En el periodo 2007 a 2010 se presentaron 264 casos sospechosos de toxocariasis, de los cuales 22 (8,3\%) tuvieron historias clínicas incompletas. Finalmente, se analizó un total de 242 pacientes, de los cuales 148 (61,2\%) fueron casos con serología positiva y 94 $(38,8 \%)$ con serología negativa.

Los pacientes estudiados presentaron una edad media de 6,7 (DE 4,0) años. Los niños mayores de 5 años fueron $144(59,5 \%)$, de sexo masculino 150 (62\%), y 149 (61,6\%) fueron atendidos en consultorio externo.

Del total de pacientes estudiados, el motivo de consulta fue eosinofilia en 159 (65,7\%). Con respecto a los exámenes de laboratorio, la media de la cuenta de leucocitos en sangre periférica fue 8908 (DE 3 278) y la hemoglobina presentó una media de 11,8 (DE 1,2) g/dL (tabla 1).

Hallamos una frecuencia de serología positiva contra toxocara de $61,2 \%$ (148 casos); los niños presentaron una edad media de 6,5 (DE 3,8) años de edad y $103(69,6 \%)$ correspondieron al grupo etario de 2 a 10 años. Fueron del sexo masculino 58 (39,2\%); $12(8,1 \%)$ mostraron algún grado de desnutrición y $105(70,9 \%)$ eosinofilia; la hemoglobina tuvo una media de $11,8 \mathrm{~g} / \mathrm{dL}$ (DE $1,2)$ y los leucocitos $9,2 \mathrm{U} \cdot \mathrm{mL}\left(10^{9} \mathrm{~L}\right)$ (DE 3,6).

\section{DISCUSIÓN}

Encontramos una frecuencia alta de $61 \%$ de serología positiva en niños con sospecha de esta enfermedad, valores muy parecidos a los comunicados por el Instituto Pedro Kouri (IPK), en Cuba, en donde hallaron frecuencias de 23\% a $59 \%$ en diferentes años ${ }^{(13)}$, en pacientes sospechosos de todas las edades. Sin embargo, podemos encontrar valores más altos en estudios realizados en Indonesia, hasta $92,8 \%^{(14,15)}$, y valores mínimos de $1,8 \%{ }^{(16)}$, como en lugares de Venezuela.

La edad promedio de los casos seropositivos fue 7 años, siendo más frecuente en los mayores de 5 años, a diferencia de otros estudios, que hallan más casos seropositivos en los niños de 1 a 4 años, por ser este grupo de edad relacionado con la pica o geofagia ${ }^{(8)}$.

Con respecto al servicio que solicitó más exámenes de ELISA para toxocara fue consultorio externo; por lo que sugerimos a los pediatras sospechar en los casos atípicos de toxocara, como exantemas hemorrágicos, convulsiones, pacientes en estudio de alergia, neumonía ${ }^{(17)}$, glomerulonefritis (18), endocarditis ${ }^{(19)}$, enfermedades neurológicas ${ }^{(20)}$.

La toxocariasis ocular es de relevancia clínica por la evolución no buena en la mayoría de los casos, pudiendo ocasionar pérdida parcial y hasta la pérdida total de la visión en el ojo afectado ${ }^{(21)}$. Es de difícil diagnóstico, por la alta frecuencia de falsos negativos en la prueba de ELISA.

La falta de diferencia entre los toxocara seropositivos y seronegativos con respecto al estado nutricional, demuestra que la patogenia de esta enfermedad no es principalmente por déficit inmunológico relacionado con la desnutrición, sino por la respuesta inmune, al ocasionar inflamación en órganos; de allí la importancia del uso de corticoides, en algunos casos.

La presencia de eosinofilia en el hemograma hace sospechar la enfermedad ${ }^{(12)}$. No obstante, esta debe ser complementada con inmunoglobulina elevada y serología positiva para toxocara, en el diagnóstico de toxocariasis visceral. Sin olvidar interrogar por la tenencia de mascotas y visita a los lugares de exposición de huevos de toxocara, como son los parques. 
Tabla 1. Análisis bivariado, factores asociados a positividad de toxocara IgG.

\begin{tabular}{|c|c|c|c|}
\hline & Negativo & Positivo & $p$ \\
\hline & $94(38,8 \%)$ & $148(61,2 \%)$ & \\
\hline Sexo (masculino) n (\%) & $34(36,2)$ & $58(39,2)$ & $0,637^{*}$ \\
\hline Edad media (DE) & $6.5(4,5)$ & $6.8(3,8)$ & $0,546^{* *}$ \\
\hline \multicolumn{4}{|l|}{ Grupo etario n (\%) } \\
\hline$<1$ año & $05(4,3)$ & $03(2,0)$ & \\
\hline $1 \mathrm{a}<2$ años & $08(8,5)$ & $04(2,7)$ & \\
\hline 2 a 5 años & $26(27,8)$ & $42(28,4)$ & \\
\hline$>5$ a 10 años & $31(33,0)$ & $61(41,2)$ & \\
\hline >10 años & $24(25,5)$ & $38(25,7)$ & $0,145^{*}$ \\
\hline \multicolumn{4}{|l|}{ Motivo de consulta } \\
\hline Eosinofilia n (\%) & $54(57,5)$ & $105(70,9)$ & $0,031^{*}$ \\
\hline Convulsiones n (\%) & $7(7,5)$ & $11(7,4)$ & $0,997^{*}$ \\
\hline Síntomas oculares n (\%) & $12(12,8)$ & $16(10,9)$ & $0,453^{*}$ \\
\hline Fiebre $n(\%)$ & $3(3,2)$ & $10(6,8)$ & $0,231^{*}$ \\
\hline Otros & 18 & 6 & \\
\hline \multicolumn{4}{|l|}{ Estado nutricional } \\
\hline Algún grado de desnutrición n (\%) & $2(2,1)$ & $12(8,1)$ & $0,052^{*}$ \\
\hline Obeso n (\%) & $7(7,5)$ & $8(5,4)$ & $0,521^{*}$ \\
\hline Sobrepeso n (\%) & $11(11,7)$ & $16(10,9)$ & $0,830^{*}$ \\
\hline \multicolumn{4}{|l|}{ Exámenes de laboratorio } \\
\hline Leucocitos (U·103·mL) media (DE) & $8,4(2,4)$ & $9,2(3,6)$ & $0,948^{* *}$ \\
\hline Leucocitosis (>1 000/mL) & $16(17,4)$ & $44(29,7)$ & $0,032 * *$ \\
\hline Eosinófilos (U·103·mL) media (DE) & $1,0(1,2)$ & $1,4(1,5)$ & $0,006^{* *}$ \\
\hline Hemoglobina $(\mathrm{g} / \mathrm{dL}$ ) media (DE) & $11,9(1,2)$ & $11,8(1,2)$ & $0,512 * *$ \\
\hline Hemoglobina $<10(\mathrm{~g} / \mathrm{dL})$ n $(\%)$ & $90(95,7)$ & $141(95,3)$ & $0,863^{*}$ \\
\hline
\end{tabular}

*chi cuadrado; ** prueba t de sudent; DE: desviación estándar.

Debemos considerar que la prueba de ELISA emplea antígenos de excreción y secreción de larvas $\mathrm{L}_{2} / \mathrm{L}_{3}$ de $\mathrm{T}$. canis, con una especificidad de 90 a $92 \%$ y sensibilidad de 75 a $86 \%{ }^{(22)}$. Por ello, debemos reconocer que la positividad de la prueba puede indicar exposición pasada y un resultado negativo no necesariamente descarta la enfermedad. Además, el resultado debe ser interpretado de acuerdo al estado clínico.

El estudio tiene varias limitaciones. Las muestras no representan toda la población de Lima y los exámenes serológicos estaban sujetos al stock de reactivos.

Por ello, sugerimos diseñar estrategias de control de esta enfermedad zoonótica por parte del personal de
Salud Ambiental y de Promoción de la Salud del Ministerio de Salud en las grandes ciudades, como Lima, por existir condiciones para convertirse en una enfermedad de importancia en la salud pública, como son las migraciones, el incremento de la población humana, incremento de animales domésticos y cambios climáticos que ayudarían al desarrollo de larvas infectantes más rápidamente ${ }^{(23,24)}$. Estas instituciones del Estado deberían hacer control poblacional canino y educación en los hábitos de higiénicos adecuados en el domicilio con mascota. También sugerimos que el personal de salud debe identificar a las personas infectadas con este parásito, dado que las lesiones graves que producen son totalmente prevenibles.
Se concluye que el Instituto Nacional de Salud del Niño atiende niños con una alta frecuencia de serología positiva contra toxocara en los pacientes sospechosos por esta enfermedad, siendo las características más importantes pertenecer al grupo de edad entre 2 y 10 años y aumento de eosinófilos en sangre periférica.

\section{REFERENCIAS BIBLIOGRÁFICAS}

1. Pelayo-Durán L. Generalidades de parasitología. En: Llop A. Microbiologia y parasitologia médica. La Habana: Editorial Ciencias Médicas; 2001.

2. Saredi N. Manual Práctico de Parasitología Médica. Buenos Aires: Laboratorio Andrómaco; 2002.

3. Beaver P, Jung RC. Parasitología Clínica. 2da. Edición ed. Barcelona: Salvat Editores; 1986.

4. Abdel Aal TM, Youssef FG, el-Hady HM, Sabry NM, Habib KS, Saber JM. Evaluation of crude and purified Toxocara canis antigens in serodiagnosis of human toxocariasis. J Egypt Public Health Assoc. 1996;71(3-4):321-41.

5. Alabiad CR, Albini TA, Santos CI, Davis JL. Ocular toxocariasis in a seronegative adult. Ophthalmic Surg Lasers Imaging. 2010 Apr 2:1-3. doi: 10.3928/15428877-20100325-06.

6. Bellanger AP, Humbert P, Gavignet B, Deschaseaux AD, Barisien C, Roussel S, et al. Comparative assessment of enzyme-linked immunosorbent assay and Western blot for the diagnosis of toxocariasis in patients with skin disorders. Br J Dermatol. 2010 Jan;162(1):80-2. doi: 10.1111/j.1365-2133. 2009.09411.x.

7. Rubinsky-Elefant G, Hirata CE, Yamamoto JH, Ferreira MU. Human toxocariasis: diagnosis, worldwide seroprevalences and clinical expression of the systemic and ocular forms. Ann Trop Med Parasitol. 2010 Jan;104(1):3-23. doi: 10.1179/136 485910X12607012373957.

8. American Academy of Pediatrics.Toxocariasis (Visceral Larva Migrans, Ocular Larva Migrans). En: Red Book: 2009 Report of the Committee on Infectious Diseases. 28th. Elk Grove Village, IL: American Academy of Pediatrics; 2009:666-7.

9. Hotez PJ, Wilkins PP. Toxocariasis: America's most common neglected infection of poverty and a helminthiasis of global importance? PLoS Negl Trop Dis. 2009;3(3):e400. doi: 10.1371/journal. pntd.0000400.

10. Fan CK, Lan HS, Hung CC, Chung WC, Liao CW, Du WY, et al. Seroepidemiology of Toxocara canis infection among mountain aboriginal adults in Taiwan. Am J Trop Med Hyg. 2004 Aug;71(2):216-21.

11. Maguiña C. Larva migrans visceral. Primer reporte en el Peru. Rev Med Hered. 199:2:14-7.

12. Roldan WH, Espinoza YA, Atuncar A, Ortega E, Martinez A, Saravia M. Frequency of eosinophilia and risk factors and their association with Toxocara infection in schoolchildren during a health survey in the north of Lima, Peru. Rev Inst Med Trop Sao Paulo. 2008 Sep-Oct;50(5):273-8.

13. Sariego I, Kanobana K, Rojas L, Speybroeck N, Polman K, Nunez FA. Toxocariasis in Cuba: a literature review. PLoS Negl Trop Dis. 2012;6(2):e1382. doi: 10.1371/journal.pntd.0001382. 
14. Chomel BB, Kasten R, Adams C, Lambillotte D Theis J, Goldsmith R, et al. Serosurvey of some major zoonotic infections in children and teenagers in Bali, Indonesia. Southeast Asian J Trop Med Public Health. 1993 Jun;24(2):321-6.

15. Magnaval JF, Michault A, Calon N, Charle JP. Epidemiology of human toxocariasis in La Reunion. Trans R Soc Trop Med Hyg. 1994 SepOct;88(5):531-3.

16. Garcia-Pedrique M. E., Diaz-Suarez O., Estevez J., Cheng-Ng R., Araujo-Fernández M., Castellano $J$. et al. [Prevalence of infection by Toxocara in schoolchildren in the community of El Mojan, Zulia state, Venezuela]. Invest Clin. 2004;45:347-54

17. Roig J, Romeu J, Riera C, Texido A, Domingo C, Morera J. Acute eosinophilic pneumonia due to toxocariasis with bronchoalveolar lavage findings. Chest. 1992 Jul;102(1):294-6.

18. Hamilton JM, Naylor J, Weatherley A. Glomerular lesions associated with infestation with Toxocara cati. Vet Rec. 1982 Dec 18;111(25-26):583-4.

19. Prunier F, Delepine S, Victor J, de Gentile L, Moreau C, Laporte J, et al. [Loffler's fibroblastic endocarditis. A report of a case complicating toxocarosis]. Arch Mal Coeur Vaiss. 2001 Mar;94(3):226-30.

20. Finsterer J, Auer H. Neurotoxocarosis. Rev Inst Med Trop Sao Paulo. 2007 Sep-Oct;49(5):279-87.

21. Shields JA. Ocular toxocariasis. A review. Surv Ophthalmol. 1984 Mar-Apr;28(5):361-81.

22. Jacquier $P$, Gottstein $B$, Stingelin $Y$, Eckert J. Immunodiagnosis of toxocarosis in humans: evaluation of a new enzyme-linked immunosorbent assay kit. J Clin Microbiol. 1991 Sep;29(9):1831-5.

23. Macpherson $\mathrm{CN}$. The epidemiology and public health importance of toxocariasis: A zoonosis of global importance. Int J Parasitol. 2013 Nov;43(1213):999-1008. doi: 10.1016/j.jpara.2013.07.004.

24. Campos Junior D, Elefant GR, de Melo e Silva EO, Gandolfi L, Jacob CM, Tofeti A, et al. [Frequency of seropositivity to Toxocara canis in children of different socioeconomic strata]. Rev Soc Bras Med Trop. 2003 Jul-Aug;36(4):509-13.

Artículo recibido el 18 de diciembre de 2013 y aceptado para publicación el 2 de mayo de 2014.

Fuente de financiamiento: autofinanciado.

Conflictos de interés:

No existen, con la publicación del estudio.

Correspondencia:

Dr. Edwin Miranda Choque

Dirección: Pachacútec 910 interior 501, Jesús María, Lima, Perú

Celular: 968544431

Correo electrónico: emirandach@hotmail.com 